\title{
Validation of Acoustic Emission (AE) Crack Detection in Aerospace Grade Steel Using Digital Image Correlation
}

\author{
R. Pullin ${ }^{1, a}$, M. J. Eaton ${ }^{1, b}$, J. J. Hensman ${ }^{2, c}$, K. M. Holford ${ }^{1, d}$, K. Worden ${ }^{2, e}$ \\ and S. L. Evans ${ }^{2, f}$ \\ ${ }^{1}$ Cardiff School of Engineering, Cardiff University, Queen's Buildings, The Parade, Cardiff, UK. \\ ${ }^{2}$ University of Sheffield, Dept. of Mechanical Engineering, Mappin Street, Sheffield, U.K.
}

\begin{abstract}
apullinr@cardiff.ac.uk, ${ }^{\mathrm{b}}$ eatonm@cardiff.ac.uk, ${ }^{c}$ james.hensman@sheffield.ac.uk, holford@cardiff.ac.uk, ${ }^{\mathrm{e}}$ k.worden@sheffield.ac.uk and 'evanssl@cardiff.ac.uk
\end{abstract}

Keywords: Acoustic emission, digital image correlation, fatigue fractures

\begin{abstract}
Acoustic Emission (AE) is a passive form of non-destructive testing that relies on the detection and analysis of stress waves released during crack propagation. AE techniques are successfully employed number of industries there remains some scepticism in aerospace engineering.

The reported investigation details a single four point bend test specimen undergoing fatigue loading. This test is part of a much larger programme designed to demonstrate a technology readiness level (TRL) of five of the use of AE to detect crack initiation and growth in landing gear structures.

The completed test required that crack growth had to be monitored to allow a comparison with the detected and located AE signals. The method of crack monitoring had to be non-contact so as not to produce frictional sources of $\mathrm{AE}$ in the crack region, preventing the use of crack mouth opening displacement gauges. Furthermore adhesives on the specimen surface had to be avoided to eliminate the possibility that the detected AE was from adhesive cracking, thus the use of strain gauges or foil crack gauges was not possible.

A method using Digital Image Correlation (DIC) to monitor crack growth was investigated. The test was stopped during fatigue loading at 1000 cycle intervals and a DIC image captured at peak load. The displacement due to crack growth was observed throughout the investigation and the results compared with the detected AE signals.

Results showed a clear correlation between AE and crack growth and added further evidence of TRL5 for detecting fractures in landing gears using AE.
\end{abstract}

\section{Introduction}

Messier-Dowty are world leaders in the design and manufacture of aircraft landing gear systems, structures and components. Each design requires certifying for flight by the airworthiness authorities and this process relies on information obtained from structural fatigue testing. Traditionally, nondestructive testing (NDT) is used to confirm the integrity of the landing gear structure at key stages in the fatigue test regime. The NDT inspection requires the test to be stopped for a period of time to allow the structure to be dismantled and inspected and these periods of NDT can account for $25 \%$ of the total testing time. It is proposed that Acoustic Emission (AE) can be used to monitor the landing gear during the certification test in order to reduce the down time associated with conventional NDT inspections.

Acoustic Emission (AE) is a passive NDT technique that relies on the detection of the stress waves that are released during crack propagation. Piezoelectric sensors coupled to a structure detect the surface stress waves. The sensors convert the surface displacements to a voltage that is sampled and based on user thresholds separated into individual discreet signals. Traditionally waveform 
parameters such as amplitude, energy and rise-time are extracted from the waveform and used for analysis.

Although AE techniques are currently employed in a number of industries, there is still some skepticism in aerospace engineering. A major aspect of implementing an AE solution for monitoring landing gears is that Messier-Dowty requires an automated system capable of identifying fractures at an early stage in what is a high-noise environment and in specimens that have complex geometry.

The reported investigation details a single specimen test that is part of a much larger programme that includes tests on real landing gears in a high noise environment, designed to demonstrate a technology readiness level (TRL) of five of using AE to detect crack initiation and growth in landing gear structures undergoing certification tests.

\section{Methodology of Developed Technique}

A methodology for automatically identifying fatigue fractures in metallic structures (Fig. 1) has been developed through an EPSRC and Messier-Dowty funded project. Full details of key aspects of the methodology have been published previously [1-6]. However, in summary, waveforms recorded using a traditional system are inputted into a novel script that extracts feature data using a fast wavelet transform. Fast wavelets are advantageous when compared to traditional AE wave descriptors which can be ambiguous. The signals are then located and clustered in the physical space (traditional location clusters). It is these clusters that form the main part of the analysis to identify fractures. Initially a measure of variance of the features of the wavelets of signals within a located cluster compared with the entire data set at a sensor is made. Signals from fractures will demonstrate a low variance in the difference in signal features as demonstrated in previous studies [5]. A further measurement of the location cluster is then made using

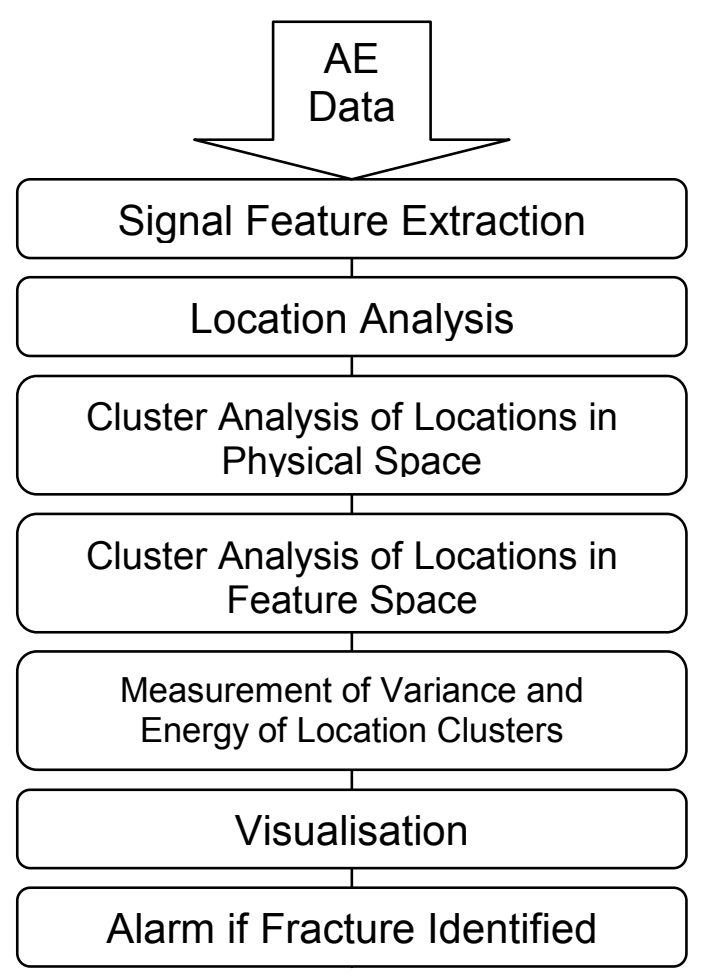

Fig. 1 Schematic of Methodology energy rates, with a rising accumulative energy trend being an indicator of fracture growth. The rising energy trend indicator is derived from the comparison of the Paris-Erdogan equation, stress intensity factors at the crack tip and crack length [7]. As crack length increases the rate of $\mathrm{AE}$ emission also increases due to the greater advances in crack length per cycle, leading to a rising energy trend. It is the low variance of data in a cluster combined with a rising energy trend that signal the onset of fracture in a specimen.

\section{Experimental Procedure}

The 4PBT specimen used for this investigation is shown in Fig. 2. The specimens were manufactured by Messier-Dowty from $20 \mathrm{~mm}$ thick aerospace grade steel. To validate the developed automated analytical techniques a digital image correlation (DIC) technique was used to monitor crack growth. During the fatigue test the cyclic loading was stopped, peak load applied and an optical measurement of the crack mouth opening was made. Using DIC offers significant advantages over foil crack gauges and a traditional crack mouth opening displacement (CMOD) gauges. Both these traditional methods can introduce acoustic emission sources into the experiment 
either through glue cracking in foil gauges or frictional noises from the CMOD contact point with the specimen.

The specimen was subjected to a fatigue load of $80 \mathrm{kN} / \mathrm{R}=0.1$ at $1 \mathrm{~Hz}$. The specimen was instrumented with four Physical Acoustics Limited (PAL) resonant frequency sensors in a rectangular array about the notch as shown in Fig. 2. Sensors were held in position with magnetic clamps and brown grease was used as a couplant. Installed sensor sensitivity was evaluated using the pencil lead fracture technique. Waveform data was recorded using a PAL PCI-2 acquisition system at sample rate of $2 \mathrm{MHz}$. All data analysis was completed post-test.

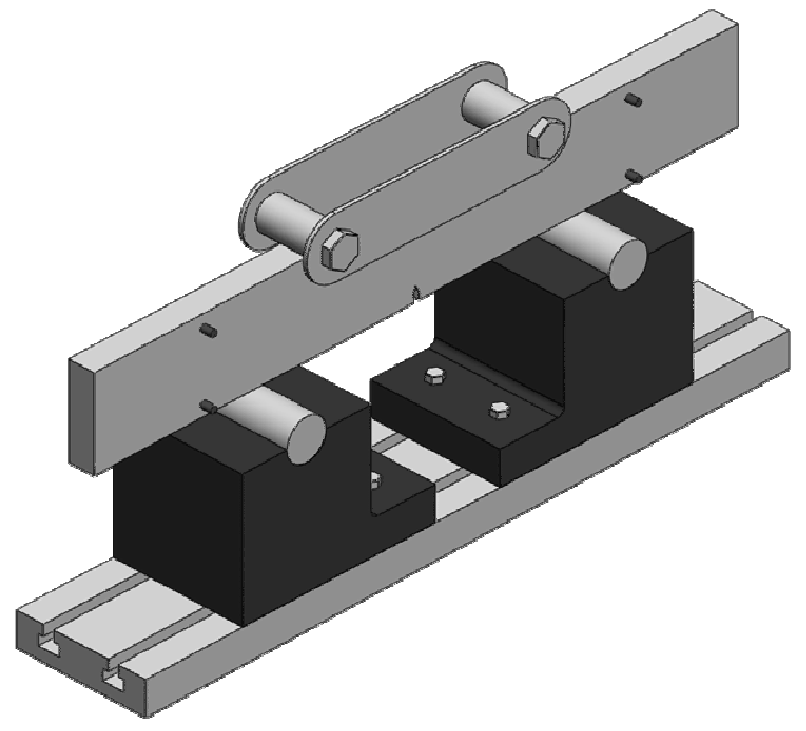

Test Specimen and Loading

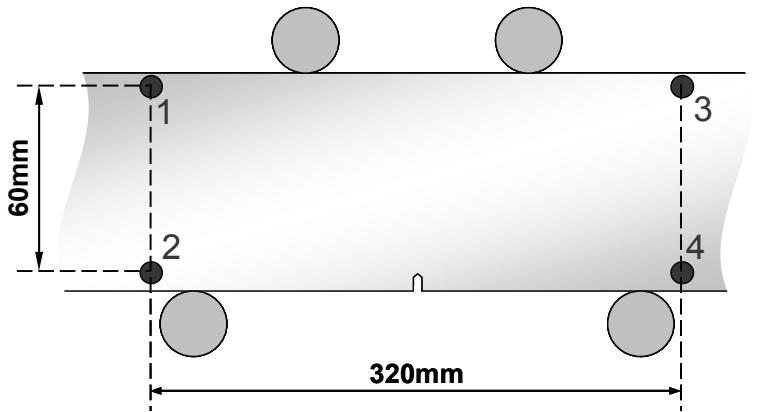

AE Instrumentation

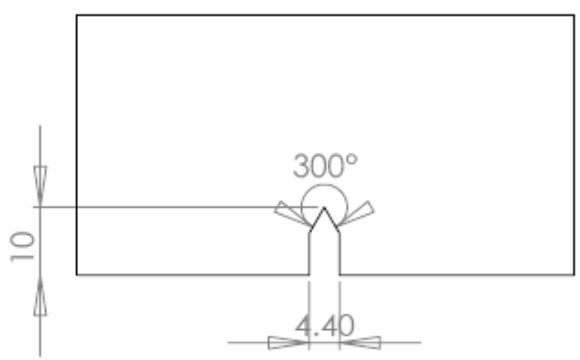

Notch Details

Fig 2. Specimen Details

\section{Results and Discussion}

Fig. 3 shows the digital image correlation CMOD measurement and AE data recorded during the fatigue test. The first DIC image was captured at $11 \mathrm{k}$ cycles and was in response to a significant increase in all detected AE energy, subsequent images were captured at $1 \mathrm{k}$ cycle intervals. It can be seen from the plot that the initial increase in acoustic activity coincides with an increase in crack mouth opening demonstrating that the AE is detecting the crack. This is further demonstrated by the location of the signals as shown in Fig 4.

The two results clearly demonstrate, in combination with the DIC CMOD measurements, the advantages and benefits of the acoustic monitoring technique. However the limited analysis completed does not provide an automated solution to detecting fatigue fractures.

Fig. 5 displays the first output of the developed script for the automatic detection of fractures. The plot shows the position of clusters, or groups, of over 40 signals in an area of $13 \times 13 \mathrm{~mm}$. The clusters are in close proximity to the notch area, acknowledging errors associated with location such as signal threshold triggering. The detected clusters all exhibit a high crack score, calculated from the negative log of the variance between features of waveforms associated with the cluster.

Previous investigations in this series of four point bend tests have demonstrated that clusters with crack scores above 28 are clear indicators of fractures with noise sources being considerably lower. In this example the specimen ruptured at $43.1 \mathrm{k}$ cycles and the initial cluster shown below occurred at approximately $8.2 \mathrm{k}$ cycles (the onset of crack growth). This coincides with CMOD data in Fig. 3. 


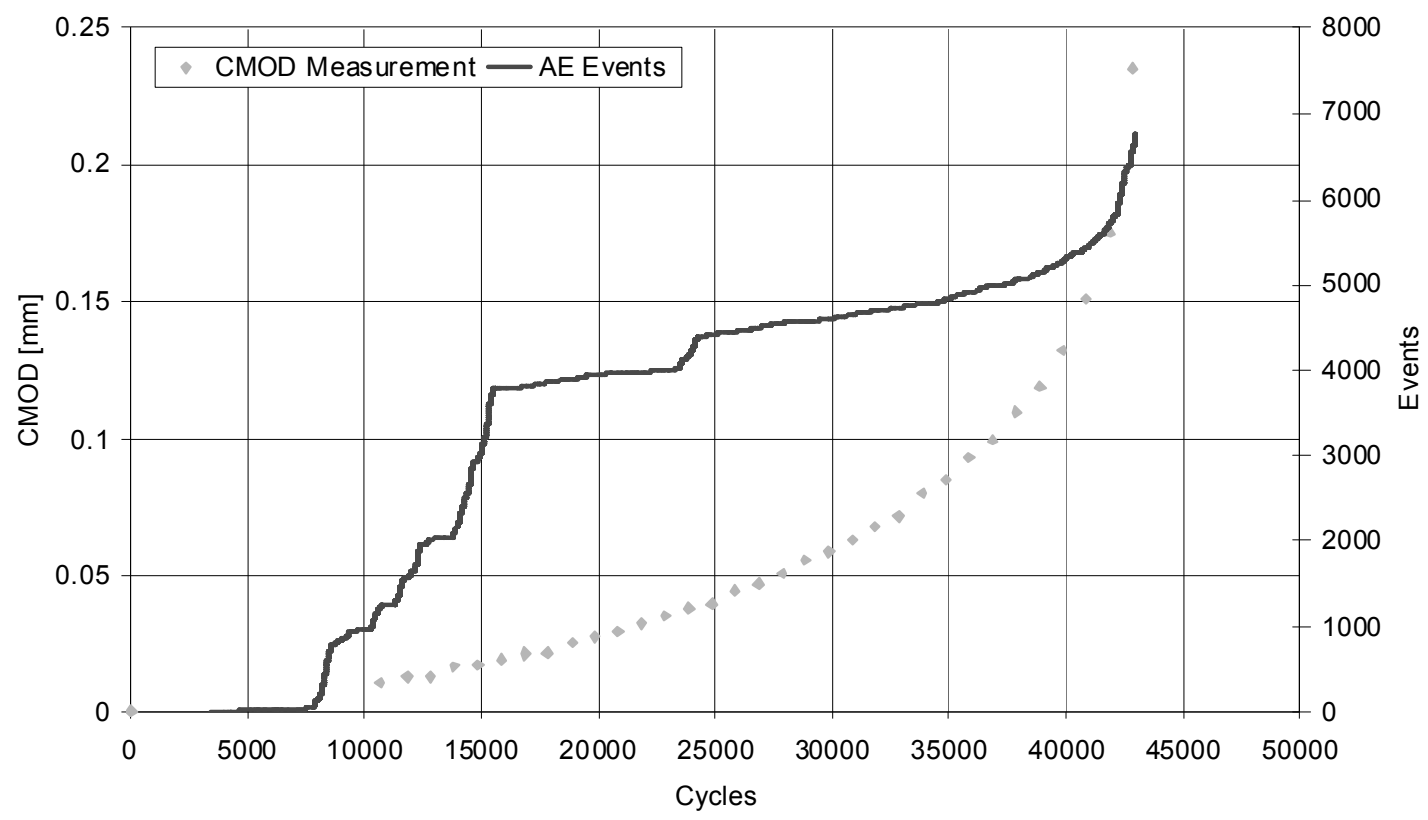

Fig 3. History of recorded DIC and AE data for duration of fatigue test

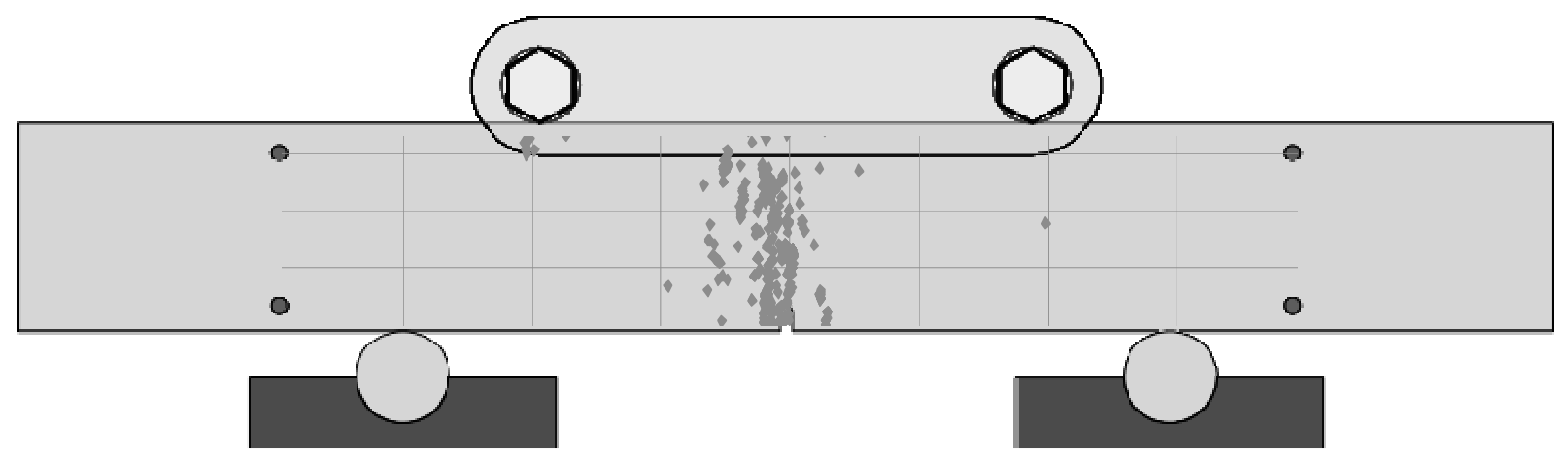

Fig 4. Location of AE signals $0-11 \mathrm{k}$ cycles

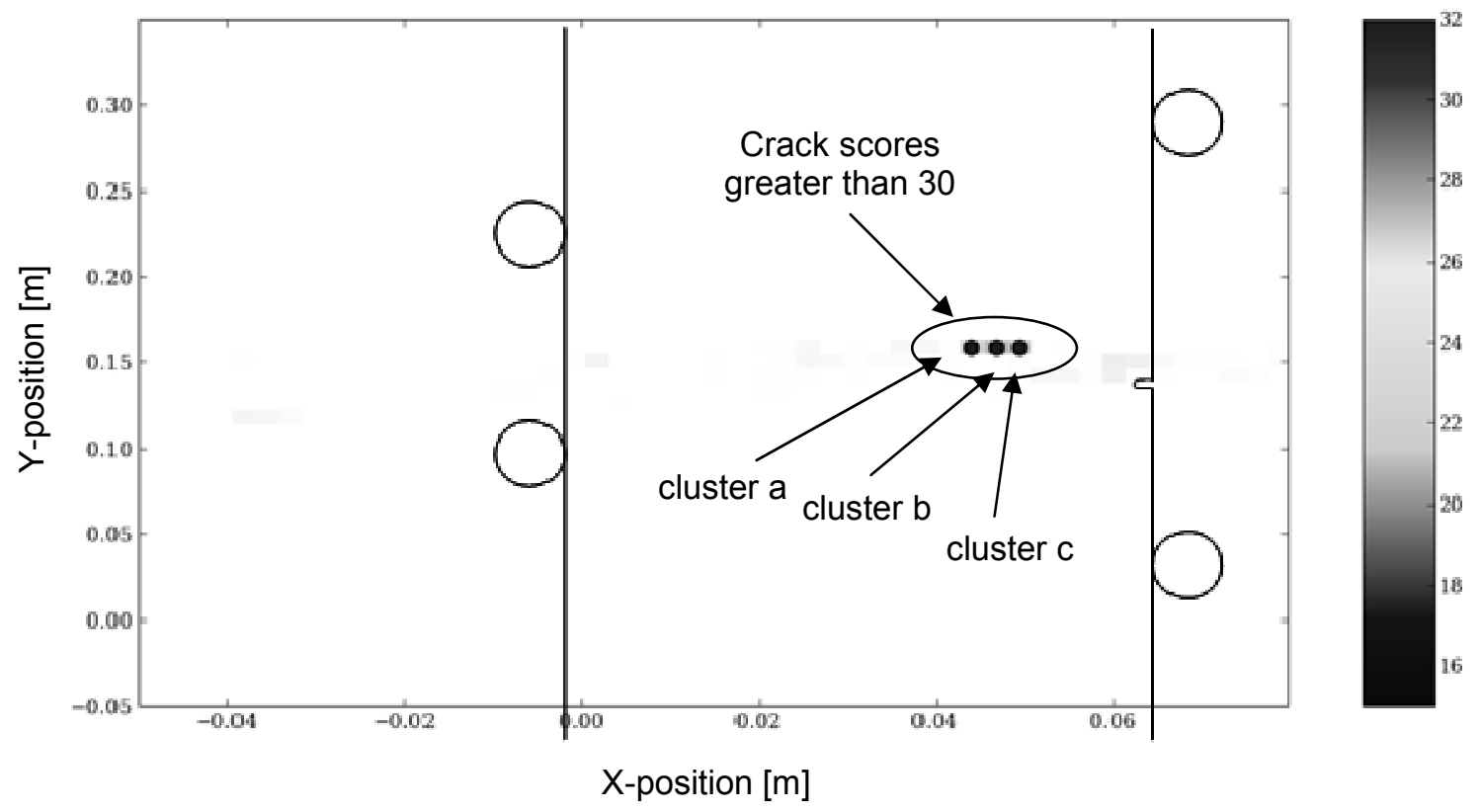

Fig. 5 Spatial and crack-score results using developed automated fracture detection script 
The second aspect of the developed work examines the cumulative energy trend of the identified clusters. As previously stated in the discussed methodology a rising energy trend is indicative of fracture source and when combined with the variance indicator an automated response to the presence of the fracture can be flagged. Fig 6 shows the energy results for the three identified clusters in Fig 5.

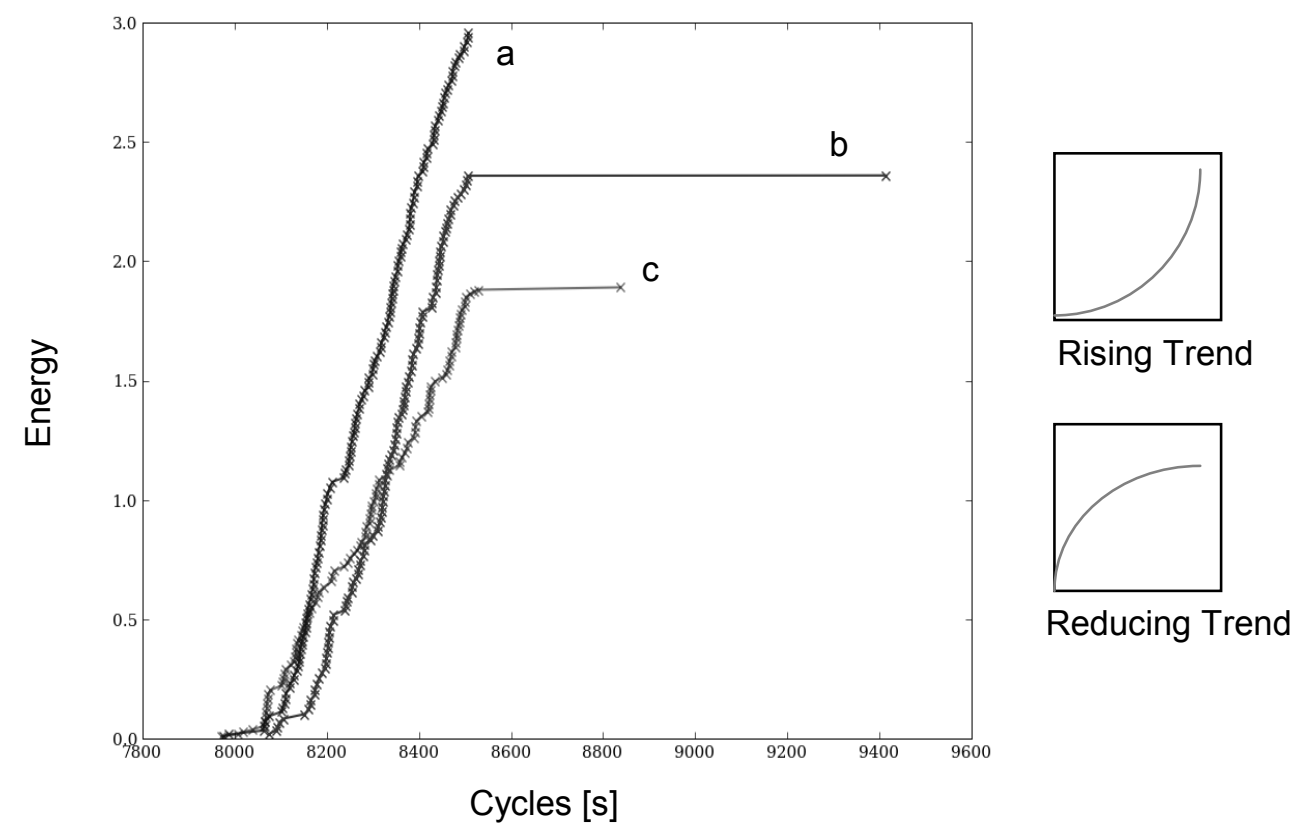

Fig. 6 Results of energy trends of spatial clusters using developed automated fracture detection script

The energy trends from the three clusters initially exhibit rising energy trends. In the script an equation for the curve is calculated mathematically and the type of curve is determined. In the example however two clusters ( $b$ and $c$ ) display a reducing trend due to signals occurring in the cluster significantly after it appears the cluster has stopped. As the fracture length increases new location clusters are created meaning earlier clusters are no longer 'live'. However if a new signal is attributed to an old 'dead' cluster the energy trend will be distorted as the cluster once again becomes 'live'. This has no bearing on the crack score. This problem is currently being investigated by introducing an online radial cluster algorithm which captures and consolidates clusters to form larger clusters which are less sensitive to additional signals being attributed to 'dead' clusters. The algorithm utilities a real time approach and reviews location clusters as they appear, hence 'online', assessing whether they are a new cluster or should be part of an existing cluster. The online radial cluster algorithm will be the focus of future publications.

The presented findings have shown that the script based on cluster variance in the feature space and energy trends would automatically indicate a fatigue fracture at $8.2 \mathrm{k}$ cycles in this specimen. The DIC image shows significant increase in CMOD at $11 \mathrm{k}$ cycles demonstrating that the fracture has started and validating the developed techniques. Results for three further investigations using four-point bend specimens are presented in Table 1 adding further validation to the developed methodology.

Further issues occur when monitoring landing gear structures undergoing certification. Landing gear structure tests are high noise environments, however noise sources have been shown to be of high variance and using an artificial source the developed techniques have been demonstrated in real tests [8]. In addition landing gears are complex structures that have a myriad of thickness changes and cutout sections which have dramatic affects on signal propagation and signal velocities which 
can considerably affect source location accuracy. This has been resolved using a touch and learn location system, called Delta-T, which has been demonstrated in a number of complex structures $[9,10]$.

Table 1: Summary of results from three further four-point bend tests

\begin{tabular}{cccccc}
\hline $\begin{array}{c}\text { Specimen } \\
\text { Number }\end{array}$ & $\begin{array}{c}\text { Position of } \\
\text { Crack Initiation } \\
{[\mathbf{m}]}\end{array}$ & $\begin{array}{c}\text { AE } \\
\text { Location } \\
{[\mathbf{m}]}\end{array}$ & $\begin{array}{c}\text { Cycles to } \\
\text { Failure } \\
{[\mathbf{k} \text { cycles] }}\end{array}$ & $\begin{array}{c}\text { Detected } \\
\text { Failure } \\
{[\text { cycles] }}\end{array}$ & $\begin{array}{c}\text { Crack } \\
\text { Score }\end{array}$ \\
\hline 1 & 0.47 & 0.47 & 1.4 & 0.9 & 28 \\
\hline 2 & 0.47 & 0.47 & 40.4 & 1.8 & 32 \\
\hline 3 & 0.45 & 0.46 & 43.7 & 10.8 & 28 \\
\hline
\end{tabular}

A series of investigations is currently being completed on a real landing gear structure with an active sliding tube creating a high noise environment to further validate the techniques. This programme of testing will be the focus of future publications.

\section{Conclusions}

The completed test has demonstrated the validity of the developed methodology. The output of the script located and identified a fracture source at approximately 8.2k cycles with final failure occurring at $43.1 \mathrm{k}$ cycles, furthermore this was confirmed using an optical CMOD measurement.

\section{Acknowledgements}

The authors would like to thank Messier-Dowty Ltd for supporting this research and the technical staff of Cardiff School of Engineering for their kind assistance with the testing programme.

\section{References}

[1] Hensman JJ et al., Society for Machine Failure Prevention Technology: 5th International Conference 15-18th July 2008

[2] Holford KM et al., $13^{\text {th }}$ International Conference on Experimental Mechanics, Greece, July $1^{\text {st }}$ $6^{\text {th }} 2007$

[3] Pullin R et al., $13^{\text {th }}$ International Conference on Experimental Mechanics, Greece, July $1^{\text {st }}-6^{\text {th }}$ 2007

[4] Hensman JJ and Worden K, $13^{\text {th }}$ International Conference on Experimental Mechanics, Greece, July $1^{\text {st }}-6^{\text {th }} 2007$

[5] Pullin R, et al., Key Engineering Materials, Vols. 347, (2007), pp. 139

[6] Roberts TM, et al., Key Engineering Materials, Vols 167-168 (1999), PP.142

[7] Pullin R, et al., Fracture Mechanics of Concrete and Concrete Structures (FRAMCOS), Italy, June $17^{\text {th }}-22^{\text {nd }} 2007$

[8] Hensman JJ, et al, Mechanical Systems and Signal Processing, Vol. 24 (2010), No. 1, pp. 211 223

[9] Baxter MG et al., Mechanical Systems and Signal Processing, Vols. 21 (2007), No. 3, pp. 1512

[10] Pullin R et al., The fifth International Conference on Acoustic Emission, Lake Tahoe, Nevada, USA, October 29 to November 2, 2007 